\title{
Resistance of superconducting nanowires connected to normal-metal leads
}

\author{
G. R. Boogaard, A. H. Verbruggen, W. Belzig,* and T. M. Klapwijk \\ Kavli Institute of Nanoscience, Faculty of Applied Sciences, Delft University of Technology, Lorentzweg 1, 2628 CJ Delft, \\ The Netherlands
}

(Received 20 January 2004; revised manuscript received 20 April 2004; published 18 June 2004)

\begin{abstract}
We study experimentally the low temperature resistance of superconducting nanowires connected to normal metal reservoirs. We find that a substantial fraction of the nanowires is resistive, down to the lowest temperature measured, indicative of an intrinsic boundary resistance due to the Andreev-conversion of normal current to supercurrent. The results are successfully analyzed in terms of the kinetic equations for diffusive superconductors.
\end{abstract}

DOI: 10.1103/PhysRevB.69.220503

PACS number(s): 74.78.Na, 74.45.+c, 85.25.Pb

Superconducting nanowires are resistive down to very low temperatures due to dynamical changes of the macroscopic phase (phase-slip). Thermally activated phase-slip (TAP) becomes more likely for reduced cross-sectional dimensions because the free-energy barrier scales with the area of the wire. Upon approaching $T \rightarrow 0$, phase slip due to thermal activation disappears and resistivity persists only by macroscopic quantum tunneling through the free-energy barrier. These processes have recently been studied in suspended carbon nanotubes coated with a thin layer of a superconducting molybdenum-germanium (MoGe) alloy, ${ }^{1,2}$ and receive increased theoretical attention. ${ }^{3,4}$ In a separate experiment ${ }^{5}$ ropes of single-walled carbon nanotubes show signs of superconductivity, which should be strongly influenced by phase-slips as well.

A second potential cause of low temperature resistance in superconducting wires is the penetration of a static electric field at normal-metal-superconductor interfaces. It reflects the conversion of current carried by normal electrons into one carried by Cooper-pairs via Andreev-reflection. It has been studied extensively close to the critical temperature, where it is related to quasiparticle charge imbalance. ${ }^{6,7} \mathrm{Al}-$ though hardly experimentally studied, at very low temperatures a similar resistive contribution is expected to be present, reflecting a length of the order of the coherence length. Since the coherence length is a sizable portion of the resistive length of the nanowires it may contribute significantly to the measured two-point resistance. Here we report experimental results on the resistance of narrow superconducting wires connected to normal metal leads (NSN, for short). We find a strong contribution to the resistance due to the conversion processes, which is analyzed and understood in terms of the nonequilibrium theory for dirty superconductors.

We chose to study samples (Fig. 1) made of superconducting (S) aluminium (Al) because of its long coherence length. Our main interest is in the two-point resistance of the $\mathrm{S}$-wire. Hence, we have chosen to work with thick and wide normal $(\mathrm{N})$ contacts with a negligible contribution to the normal state resistance. To minimize interface resistances due to electronic mismatch of both materials, we have chosen to work for $\mathrm{N}$ with bilayers of aluminium covered with thick normal metal $(\mathrm{Cu})$. In such a geometry the supercon- ducting aluminium wire is directly connected to normal aluminium.

The S-wire of $100 \mathrm{~nm}$ thick $\mathrm{Al}$ is made by evaporating $99.999 \%$ purity $\mathrm{Al}$ at a rate of $\sim 1 \mathrm{~nm} / \mathrm{s}$ in a vacuum of 1 $\times 10^{-8}$ mbar during evaporation. Films made in the same way, have a residual resistance ratio, $\mathrm{RRR}=R_{300 \mathrm{~K}} / R_{4.2 \mathrm{~K}}$ of 7.5 indicative of the level of impurity scattering. Taking the phonon resistivity of $\rho_{\mathrm{ph}}(\mathrm{Al})=2.7 \mu \Omega \mathrm{cm}$, the impurity resistivity is $\rho_{0}=0.4 \mu \Omega \mathrm{cm}$. Using $\sigma_{0}=N(0) e^{2} D$ and renormalized free-electron parameters: $N(0)=2.2 \times 10^{47} \mathrm{~J}^{-1} \mathrm{~m}^{-3}$ and $v_{F}=1.3 \times 10^{6} \mathrm{~m} / \mathrm{s}$ (Ref. 8), we find the elastic mean free path $\ell$ of $100 \mathrm{~nm}$, presumably limited by the thickness. The superconducting transition temperature of the $100 \mathrm{~nm}$ film is $1.26 \mathrm{~K}$, the usually enhanced value for aluminium thin films.

The sample is made in one evaporation run using shadow evaporation. A $300 \mathrm{~nm}$ PMMA/500 nm PMMA-MAA liftoff mask defines the width and the length of the S-wire. The width is approximately $250 \mathrm{~nm}$ and the length is varied from $1 \mu \mathrm{m}$ to $4 \mu \mathrm{m}$. The wire and the aluminium of the normal reservoirs is deposited in one run, both $100 \mathrm{~nm}$ thick. The reservoirs are made normal by a second evaporation of $470 \mathrm{~nm}$ copper $(\mathrm{Cu})$ on top. The $\mathrm{Cu}$ is evaporated at a rate of $\sim 2.5 \mathrm{~nm} / \mathrm{s}$ at a pressure of $1 \times 10^{-7} \mathrm{mbar}$. The materialparameters are $\rho_{0}=0.4 \mu \Omega \mathrm{cm}, \quad \ell=165 \mathrm{~nm}, \quad D=66$

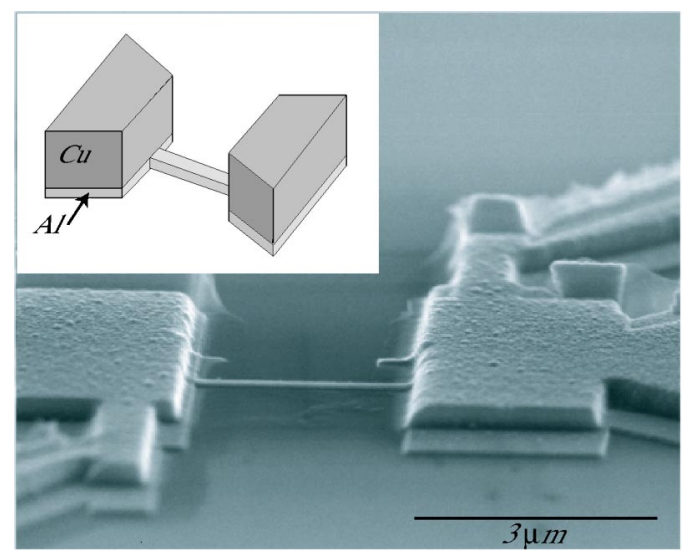

FIG. 1. SEM picture of a device (slightly misaligned), showing the coverage of the thin aluminium film with the thick $\mathrm{Cu}$ layer (except for one the measured devices are carefully lined up). The inset shows a schematic picture of an ideal device. 


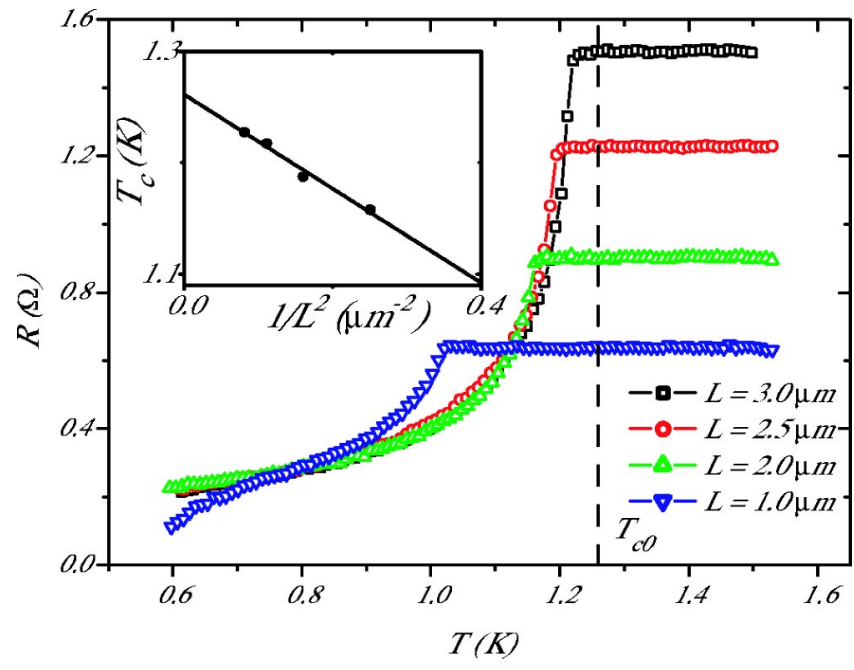

FIG. 2. (Color online) Measured $R-T$ curves for four different bridge lengths. The intrinsic $T_{c 0}$ is indicated by the vertical dashed line. The inset shows the measured critical temperature of the wire versus $1 / L^{2}$, which is used to determine $T_{c 0}$ by letting $L \rightarrow \infty$.

$\times 10^{-3} \mathrm{~m}^{2} / \mathrm{s}$ using $N(0)=1.5 \times 10^{47} \mathrm{~J}^{-1} \mathrm{~m}^{-3}$, and $v_{F}=1.2$ $\times 10^{6} \mathrm{~m} / \mathrm{s}$. The nonsuperconducting properties of the final reservoirs are confirmed by measuring the resistance of a $100 \mathrm{~nm} \mathrm{Al} / 470 \mathrm{~nm} \mathrm{Cu}$ bilayer down to the lowest temperature measured: $600 \mathrm{mK}$. No sign of superconductivity is observed. This is in agreement with the analysis of $T_{c}$ for a bilayer using the Usadel equations. ${ }^{9}$ Only for a transparency of the $\mathrm{Al} / \mathrm{Cu}$ interface lower than 0.1 the $T_{c}$ would reach values above $600 \mathrm{mK}$. 0.1 is an unrealistically low transparency for an in vacuum prepared $\mathrm{Al} / \mathrm{Cu}$ interface.

The two-point resistance is probed with a current of $1 \mu \mathrm{A}$ (linear regime) in a ${ }^{3} \mathrm{He}$ system down to $600 \mathrm{mK}$. The voltage over the wire is measured with a lock-in amplifier at $133 \mathrm{~Hz}$.

We find that the normal state resistance of nominally identical wires scatters substantially ( $10 \%$ or more), presumably due to grain-sizes compared to wire-widths. To allow a quantitative evaluation we have selected a set of wires with identical values of $\mathrm{RRR}=3.2 \pm 0.1$. Figure 2 shows the $R-T$ measurements for this particular set of wires. Samples with different RRR values show qualitatively identical behavior. All wires show a finite remaining resistance as most striking result. ${ }^{10}$

Obviously, despite the differences in length, the resistances at low temperatures have identical values and follow the same trace. It indicates that the origin of this remaining resistance is likely due to the region in the S-wire next to the interface with the normal reservoir. The resistance at $600 \mathrm{mK}$ is equal to a normal segment of the superconductor with a length of about $200 \mathrm{~nm}$.

Figure 2 (inset) also shows that the critical temperature of the wire decreases linearly with increasing the inverse square of the wire length.

The critical temperature of the wire should follow a straightforward Ginzburg-Landau analysis. For $\xi_{\mathrm{GL}}(T) \leqslant \pi L$ the bridge should become superconducting. This leads to an $\sim 1 / L^{2}$ onset-temperature according to

$$
\frac{T_{c}}{T_{c 0}}=1-2.2 \pi \frac{\hbar D}{\Delta(0)} \frac{1}{L^{2}}
$$

For $L \rightarrow \infty$ the normal contact can no longer depress $T_{c}$ and we find the intrinsic critical temperature. For the studied wires it is found to be $T_{c 0}=1.26 \mathrm{~K}$, identical to the independently determined values of the $100 \mathrm{~nm} \mathrm{Al} \mathrm{film}$.

From the normal state resistance we infer an impurity resistance of $\rho_{0}=1.1 \mu \Omega \mathrm{cm}$, in accordance with the RRR $=3.2$. It is significantly higher than the $\rho_{0}$ of the $100 \mathrm{~nm} \mathrm{Al}$ film, leading to a lower diffusivity and elastic mean free path: $D=160 \mathrm{~cm}^{2} / \mathrm{s}$, and $\ell=37 \mathrm{~nm}$, respectively. The resulting coherence length $\xi=\sqrt{\hbar D / 2 \pi k_{B} T_{c}}=124 \mathrm{~nm}$.

Finally, we estimate a resistance contribution of $11 \mathrm{~m} \Omega$ due to the spreading resistance in the normal reservoirs at low temperatures, considerably less than the value we measure in Fig. 2.

Theoretically, since the studied nanowires show diffusive transport, the Usadel equations should apply to the system. ${ }^{11}$ It is most convenient to calculate the normal current for a given applied voltage difference, assuming linear response. Schmid and Schön ${ }^{12}$ have shown that within this limit the normal current can be described with a variation, $\delta f(E, x)$, in the electronic distribution function $f(E, x)$ :

$$
I=\frac{A \sigma}{e} \int_{-\infty}^{+\infty} M(E, 0) \frac{\partial \delta f(E, 0)}{\partial x} d E
$$

with $x$ the coordinate along the length of the wire. $\delta f(E, x)$ is determined from a Boltzmann-equation, which includes the conversion of electrons into Cooper-pairs, but ignores inelastic electron-phonon scattering (only relevant close to $T_{c}$ ):

$$
\hbar D \frac{\partial}{\partial x}\left(M \frac{\partial \delta f}{\partial x}\right)-2 \Delta N_{2} \delta f=0 .
$$

The position-dependent spectral conductivity $M(E, x)$ consist of two parts $N_{1}(E, x)=\operatorname{Re}(G)$ and $N_{2}(E, x)=\operatorname{Re}(F)$, with $G$ the normal and $F$ the anomalous Greens function:

$$
M(E, x)=\left(N_{1}(E, x)\right)^{2}+\left(N_{2}(E, x)\right)^{2},
$$

$N_{1}(E, x)$ is comparable to the standard BCS density of states. The applied voltage $V$, is taken into account via the boundary conditions for Eq. (3),

$$
\delta f(E ; 0, L)=\frac{ \pm e V / 2}{4 k_{B} T \cosh ^{2}\left(E / 2 k_{B} T\right)} .
$$

Hence, the normal metal leads are taken as equilibrium reservoirs. The strength of the pairing interaction $\Delta(x)$ in Eq. (3) is determined by solving the Usadel equation in the Matsubara representation:

$$
\begin{gathered}
\frac{1}{2} \hbar D \frac{\partial^{2} \theta}{\partial x^{2}}=-\Delta(x) \cos \theta+\omega_{n} \sin \theta \\
\omega_{n}=(2 n+1) \pi k_{B} T, \quad n=0,1, \ldots
\end{gathered}
$$

in conjunction with the self-consistency equation 


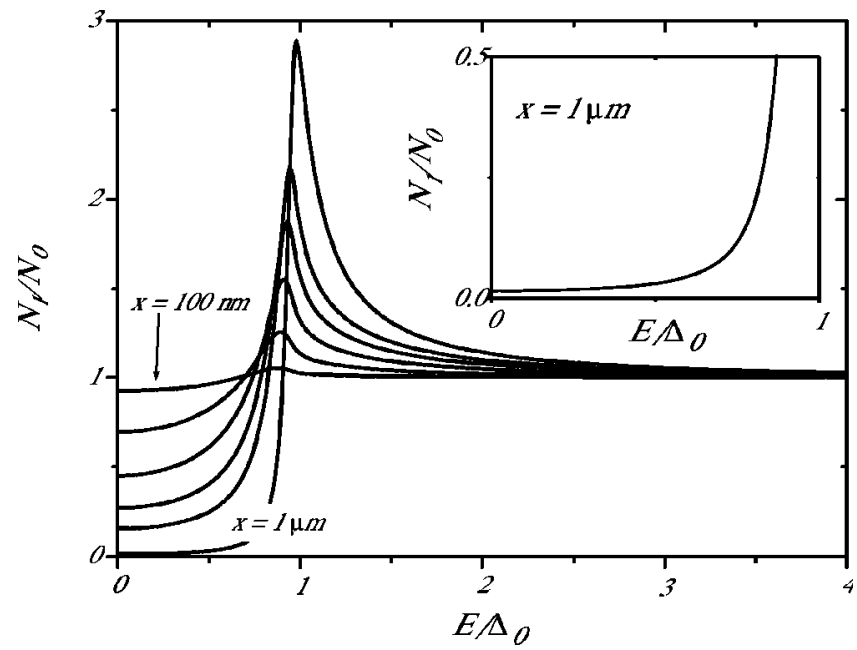

FIG. 3. The calculated density-of-states $N_{1}$ at various distances from the reservoirs $(x=100,200,300,400,500,1000 \mathrm{~nm})$ for a $2 \mu \mathrm{m}$ long wire $\left(t=0.4, D=160 \mathrm{~cm}^{2} / \mathrm{s}\right.$, and $\left.\Delta_{0}=192 \mu \mathrm{eV}\right)$. Note the exponentially small but finite subgap density-of-states in the middle (at $x=1 \mu \mathrm{m}$; see inset).

$$
\Delta \ln \left(\frac{T_{c}}{T}\right)=2 \pi k_{B} T \sum_{n=0}^{\infty}\left(\frac{\Delta}{\omega_{n}}-\sin (\theta)\right) .
$$

The so-called proximity angle, $\theta$, parametrizes the normal Green's function $G=\cos \theta$ and the anomalous Green's function $F=\sin \theta$. The spectral functions are calculated by again solving the Usadel equation but now for $\omega \rightarrow-i E$. The large normal metal reservoirs impose the boundary condition $\theta(x$ $=0, L)=0$

Our main interest is the question how the current conversion process contributes to the resistance. First of all, the presence of decaying normal electron states suppresses the gap in the density of states.

In Fig. 3, the density-of-states $N_{1}(E)$ is given for several positions along the wire of $L=2 \mu \mathrm{m}, D=160 \mathrm{~cm}^{2} / \mathrm{s}, \Delta_{0}$ $=192 \mu \mathrm{eV}$, and $T / T_{c}=0.4$. Clearly, moving away form the normal leads the density of states resembles more and more the well-known BCS density of states. Note however, that a finite subgap value remains in the middle $(x=1 \mu \mathrm{m})$ even for very long wires. This is an intrinsic result for any NSN system and it is not due to current-flow, since this result is calculated in thermal equilibrium. (The back-action of the current-flow on the spectral properties can be neglected in the linear response regime.)

In Fig. 4, we show the results of a calculation of the voltage as a function of position along the wire for two different temperatures: $t=0.4$, and $t=0.9$ with $D=160 \mathrm{~cm}^{2} / \mathrm{s}$, and $\Delta_{0}=192 \mu \mathrm{eV}$. At the temperature close to the transition temperature, the electric field penetrates the sample completely and the resistance is close to the normal state value. At low temperatures, the electric field still penetrates the superconductor over a finite length, leaving a middle piece with hardly any voltage drop. ${ }^{13}$ The penetration length is of the order of the coherence length. The inset shows the position dependent normal currents and supercurrents illustrating the current conversion processes.

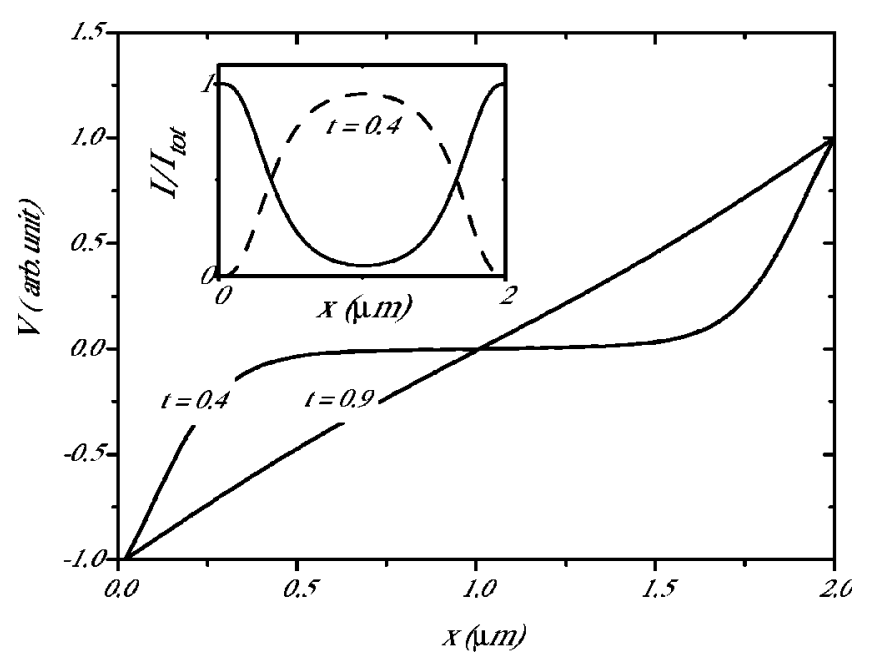

FIG. 4. The voltage in the superconducting wire as a function of position for two different temperatures $(t=0.4$ and 0.9$)$. At $t=0.9$ the wire behaves as a normal metal and for $t=0.4$ the voltage is clearly present to a depth $\xi$ (wire length $2 \mu \mathrm{m}$ with $D$ $=160 \mathrm{~cm}^{2} / \mathrm{s}$ and $\left.\Delta_{0}=192 \mu \mathrm{eV}\right)$. The inset shows the position dependent normal currents and supercurrents.

In Fig. 5, a comparison is made between the calculated resistance as a function of temperature and the measurement for a $L=2 \mu \mathrm{m}$ wire. The calculation is done with parameters $D=160 \mathrm{~cm}^{2} / \mathrm{s}$, as determined from the impurity resistivity, and $\Delta(0)=1.764 k_{\mathrm{B}} T_{c}=192 \mu \mathrm{eV}$ with $T_{c}=1.26 \mathrm{~K}$ determined from the length dependence. These parameters have been determined independently. Without any fitting parameter, the agreement between the model (dots) and the experiment (data points: open symbols) is encouragingly good. Only at the lower temperatures the observed resistance is slightly less than the theoretically predicted values.

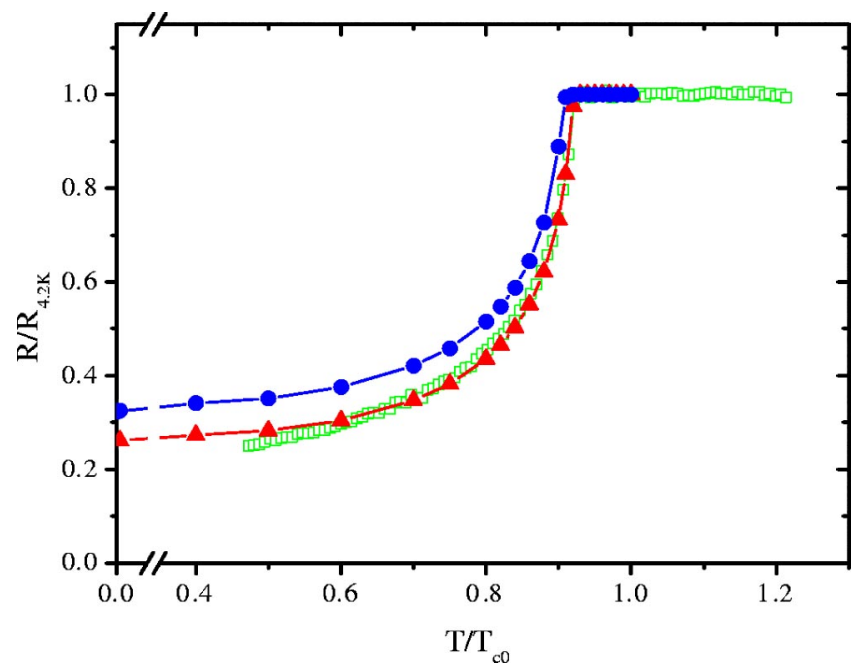

FIG. 5. (Color online) The measured $R-T$ curve for the $2 \mu \mathrm{m}$ bridge together with the model calculation using the boundary condition $\theta(x=0, L)=0$ (dots), and using the boundary condition $\partial_{x} \theta(x=0, L)=\theta(x=0, L) / a$ (triangles) with $a=75 \mathrm{~nm}$. The value for $T=0$ is 0.3255 for the hard boundary conditions (dots), and 0.2609 for the soft boundary conditions. 
Apparently, the model is overestimating the remaining resistance below $T=0.8 \mathrm{~K}$. Since there is little freedom left, we have very few options to remedy this discrepancy. The most likely option is that the rigid boundary conditions imposed at the interfaces should be relaxed. There is a finite possibility for superconducting correlations to extend into the normal metal reservoirs, which would mean that the boundary condition $\theta(x=0, L)=0$ is too rigid. Since the correlations will extend into a three-dimensional volume we assume that using the boundary conditions $\partial_{x} \theta(x=0, L)=\theta(x=0, L) / a$, i.e., a decay over a fixed characteristic length $a$, is a realistic assumption. It assumes a geometric dilution of the correlations. The result is shown in Fig. 5 by filled triangles. The best agreement between measurement and calculation model is obtained for $a=75 \mathrm{~nm}$. This value appears reasonable for a decay length since it is comparable to the dimensions $(100 \mathrm{~nm} \times 250 \mathrm{~nm})$ of the wire which emits into the reservoirs. The shortest bridge shown in Fig. 2 is found to have a further reduction in resistance, which we attribute to a small misalignment as shown in Fig. 1. Note however that the model predicts that NSN devices will continue to be resistive down to $T=0 \mathrm{~K}$. For the rigid boundary conditions we find $R / R_{4.2 K}=0.3255$ and for the soft boundary conditions we find $R / R_{4.2 \mathrm{~K}}=0.2609$.

An early indication of the low temperature boundary reported here is given by Harding et al. ${ }^{14}$ They studied the resistance of thick sandwiches of $\mathrm{Pb}-\mathrm{Cu}-\mathrm{Pb}$. By subtracting the contribution to the resistance of the $\mathrm{Cu}$-layers they identified a remaining boundary resistance which depended on the mean free path in the Pb layers. In later work by Hsiang and Clarke $^{7}$ such a resistance appeared to be unobservable, in contrast however to more recent work by Gu et al. ${ }^{15}$ The geometry of our sample, with a negligible contribution to the resistance from the reservoirs, allows us to measure only the resistance due to the conversion processes in the superconductor. In response to the work of Harding et al. Krähenbühl and Watts-Tobin ${ }^{16}$ derived an analytical expression for the effective length of the boundary resistance: $x_{0}=\sqrt{\frac{1}{6} \pi \xi_{0} l} f(T)$ with $\ell$ the mean free path for elastic scattering, $\xi_{0}$ the BCS coherence length, and $f$ a function of temperature. For $T$ $=0 \mathrm{~K}$, the function $f$ is of order 1 . In contrast to our model Ref. 16 allows for one-dimensional diffusion in $N$, rather than treating $N$ as an equilibrium reservoir.

In conclusion, we have shown that the resistance of a superconducting nanowire connected to normal leads has a finite dc resistance down to very low temperatures. The microscopic theory describes the data very well and provide a detailed image of the conversion from normal current into supercurrent, over a few coherence lengths. The results emphasize the important role of the length of the wires in relation to the nature of the contacts. ${ }^{3}$ It also explains results obtained with diffusion-cooled hot-electron bolometers in which normal leads are used to provide rapid out-diffusion of hot electrons from a superconducting wire. ${ }^{10}$

We thank the Stichting voor Fundamenteel Onderzoek der Materie (FOM) for financial support. We thank R. S. Keizer and A. A. Golubov for stimulating and clarifying discussions.
*Current address: Department of Physics and Astronomy, University of Basel, Klingelbergstrasse 82, CH-4056 Basel, Switzerland.

${ }^{1}$ A. Bezryadin, C. N. Lau, and M. Tinkham, Nature (London) 404, 971 (2000).

${ }^{2}$ C. N. Lau, N. Markovic, M. Bockrath, A. Bezryadin, and M. Tinkham, Phys. Rev. Lett. 87, 217003 (2001).

${ }^{3}$ S. Sachdev, P. Warner, and M. Troyer, Phys. Rev. Lett. 92, 237003 (2004).

${ }^{4}$ H. P. Büchler, V. B. Geshkenbein, and G. Blatter, Phys. Rev. Lett. 92, 067007 (2004).

${ }^{5}$ M. Kociak, A. Yu. Kasumov, S. Guéron, B. Reulet, I. I. Khodos, Yu. B. Gorbatov, V. T. Volkov, L. Vaccarini, and H. Bouchiat, Phys. Rev. Lett. 86, 2416 (2001); A. Kasumov, M. Kociak, M. Ferrier, R. DeBlock, S. Guéron, B. Reulet, I. Khodos, O. Stéphan, and H. Bouchiat, Phys. Rev. B 68, 214521 (2003).

${ }^{6}$ J. Clarke, Phys. Rev. Lett. 28, 1363 (1972).

${ }^{7}$ T. Y. Hsiang and J. Clarke, Phys. Rev. B 21, 945 (1980).

${ }^{8}$ N. W. Ashcroft and N. D. Mermin, Solid State Physics (Saunders College, New York, 1976).

${ }^{9}$ J. Aarts, J. M.E. Geers, E. Brück, A. A. Golubov, and R. Coehoorn, Phys. Rev. B 56, 2779 (1997), Appendix.
${ }^{10}$ I. Siddiqi, A. Verevkin, D. E. Prober, A. Skalare, B. S. Karasik, W. R. McGrath, P. Echternach, and H. G. LeDuc, IEEE Trans. Appl. Supercond. 11, 958 (2001); A. H. Verbruggen, T. M. Klapwijk, W. Belzig, and J. R. Gao, in 12th International Symposium on Space TeraHertz Technology, edited by Imran Mehdi (JPL Publications, San Diego, 2002), p. 42.

${ }^{11}$ The Usadel equations assume diffusive scattering, whereas in our wires the variation in RRR suggests that the scattering throughout the wire might be inhomogeneous, an effect we will further ignore.

${ }^{12}$ A. Schmid and G. Schön, J. Low Temp. Phys. 20, 207 (1975).

${ }^{13}$ This would be the voltage measured with a normal probe, which exchanges quasiparticles, compared to a superconducting probe, which measures the chemical potential of the superconducting condensate.

${ }^{14}$ G. R. Harding, A. B. Pippard, and J. R. Tomlinson, Proc. R. Soc. London, Ser. A 340, 1 (1974).

${ }^{15}$ J. Y. Gu, J. A. Caballero, R. D. Slater, R. Loloee, and W. P. Pratt, Jr., Phys. Rev. B 66, 140507(R) (2002).

${ }^{16}$ Y. Krähenbühl and R. J. Watts-Tobin, J. Low Temp. Phys. 35, 569 (1979). 